\title{
Nail psoriasis - what a rheumatologist should know about
}

\author{
Barbara Nieradko-Iwanicka \\ Chair and Department of Hygiene, Medical University of Lublin, Poland
}

\begin{abstract}
Psoriasis is a chronic recurrent inflammatory skin disease with prevalence of $1-3 \%$. Nail psoriasis affects $10-90 \%$ of patients with plaque psoriasis.

The aim of the article is to review the literature for the correlation between nail psoriasis and psoriatic arthritis (PsA) to provide rheumatologists a short review on features of nail psoriasis, methods of their assessment and possible clinical repercussions.

The PubMed database was searched using the key words 'nail psoriasis' and 'psoriatic arthritis'. Psoriasis involving the nail matrix shows up as changes such as pitting, Beau lines, leukonychia, red spots in the lunula, or nail plate crumbling. Nail bed psoriasis manifests as onycholysis, oil drops (or salmon patches), dyschromia, splinter hemorrhages, or subungual hyperkeratosis. Nail psoriasis and psoriatic lesions in the gluteal cleft and on the scalp usually accompany PsA, especially in adult men.
\end{abstract}

Key words: psoriasis, nail psoriasis, psoriatic arthritis.

\section{Introduction}

Psoriasis is a chronic recurrent inflammatory skin disease with prevalence of 1-3\% [1]. Nails are skin appendages. Nail psoriasis affects $10-90 \%$ of adult patients with plaque psoriasis, and has been reported in 63-83\% of patients with psoriatic arthritis (PsA) [2, 3]. In children with psoriasis the prevalence of nail involvement is $32.3 \%$ [4]. Nail involvement in psoriatic patients has a significant impact on their quality of life. Such nails are painful and cause restrictions in patients' daily activities. As the fingernails grow $0.1 \mathrm{~mm} /$ day, psoriatic nail lesions can be troublesome over a long time. Nail psoriasis, psoriatic lesions in the gluteal cleft and on the scalp usually accompany PsA, especially in adult men $[5,6]$. About $50 \%$ of patients with nail psoriasis complain of pain, stiffness or swelling of the interphalangeal joint in the absence of a diagnosis of arthropathy, which could suggest the early stage of PSA [7]. The triggering factors of nail psoriasis can be repeated trauma (in manual workers), stress, infection, and use of lithium, $\beta$-blockers or interferon [5]. Nail psoriasis is accompanied by onychomycosis in up to $27 \%$ of cases. Therefore direct examination with potassium chloride and fungal culture prior to treatment initiation are recommended [5].
The aim of the article is to review the literature for the correlation between nail psoriasis and psoriatic arthritis (PsA) to provide rheumatologists a short review on features of nail psoriasis, methods of their assessment and possible clinical repercussions.

The PubMed database was searched using the key words 'nail psoriasis' and 'psoriatic arthritis'.

\section{Results and discussion}

In a healthy nail the nail matrix is visible as white lunula [8]. If psoriasis involves the nail matrix, nail psoriasis shows up as changes in the nail plate such as pitting, Beau lines, leukonychia, red spots in the lunula, or nail plate crumbling. Nail pits are small depressions in the surface of the nail plate. Beau lines appear when the proximal or intermediate matrix is affected along its length. Leukonychia are small smooth white areas in the nail plate resulting from formation of deeper pits. Crumbling refers to large, depressed, rough areas of the nail plate, sometimes described as confluent pitting. Red dots in the lunula are red areas in the white half-moon area of the nail [5].

The nail bed is distal to the nail matrix, appears pink and lies under the translucent nail plate. Nail bed pso- 
riasis manifests as onycholysis, oil drops (or salmon patches), dyschromia, splinter hemorrhages, and subungual hyperkeratosis. Onycholysis is separation of the nail plate from the underlying nail bed. Oil drops (or salmon patches) is reddish-brown discoloration of the nail bed. Splinter hemorrhages are thin longitudinal dark brown streaks under the nail plate, but they are not specific for psoriasis [9]. The proximal and lateral nail folds, hyponychium (the area under the distal edge of the nail plate) and cuticle can all be affected by psoriasis. Other forms of nail psoriasis can be paronychia and acropustulosis. Paronychia is associated with erythematous scaly lesions affecting the proximal and/or lateral edge of the nail alongside the matrix or nail plate lesions. Acropustulosis presents with periungual or subungual pustules in patients with acrodermatitis continua of Hallopeau or pustular psoriasis [5] (Figs. 1, 2).

The Group for Research and Assessment of Psoriasis and Psoriatic Arthritis (GRAPPA) recommends the Nail Psoriasis Severity Index (NAPSI) as a grading scale for assessment of nail psoriasis. NAPSI is a numeric, objective, quantitative, simple and quick tool for evaluation of nail psoriasis. It grades all ten fingernails. NAPSI grades the severity of the nail bed and nail matrix by area of invoIvement in the nail unit [10]. Its modifications are also used [11-13]. For NAPSI evaluation the nail is divided into 4 quadrants. Each quadrant is evaluated for the features of nail bed psoriasis and nail matrix psoriasis. The nail matrix involvement is graded $0-4$ for pitting, leukonychia, red spots in the lunula and crumbling. The nail bed is also graded $0-4$ for onycholysis, splinter hemorrhages, subungual hyperkeratosis and oil drops. There is a significant correlation between target NAPSI score and evidence of enthesopathy in the course of PSA [14].

In PsA the Physician Global Assessment (PGA) scale is recommended by Outcome Measures in Rheumatology Clinical Trials (OMERACT) together with peripheral joint activity, skin activity, pain, physical function, and health-related quality of life. Several other domains (spinal disease, dactylitis, enthesitis, fatigue, nail disease, radiography, physician global assessment, and acute-phase reactants) are considered important [15].

Nail PGA evaluation grades from 0 to 4 (0 clear, 1 almost clear, 2 mild, 3 moderate, 4 severe) the proximal and distal nail matrix as well as the nail bed (in \%). For proximal nail matrix PGA the total number of lesions in the proximal nail plate is assessed: clear if there are 2 or fewer leukonychia or surface defects other than pits; almost clear if there are 1 to 5 pits/leukonychia or red spots in the lunula; mild if there are 6 to 10 pits, leukonychia or red spots; moderate if there are 11 to 20 pits, leukonychia or red spots; and severe if there are 21 or more pits, leukonychia red spots or crumbling in

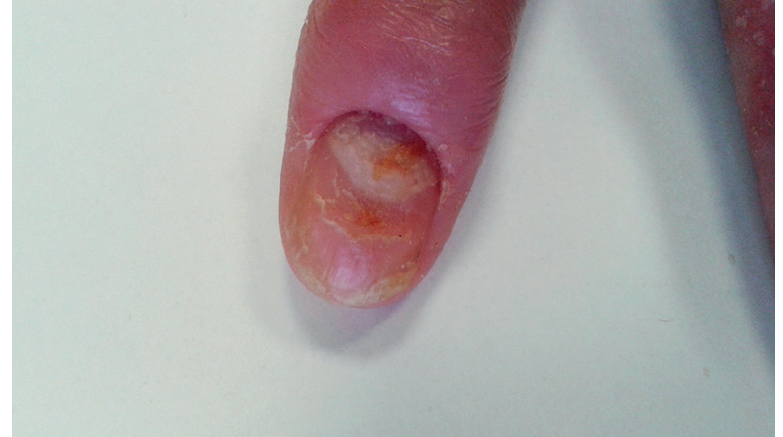

Fig. 1. Nail psoriasis with lesions in the nail matrix (leukonychia and nail plate crumbling), nail bed (onycholysis) and paronychia.

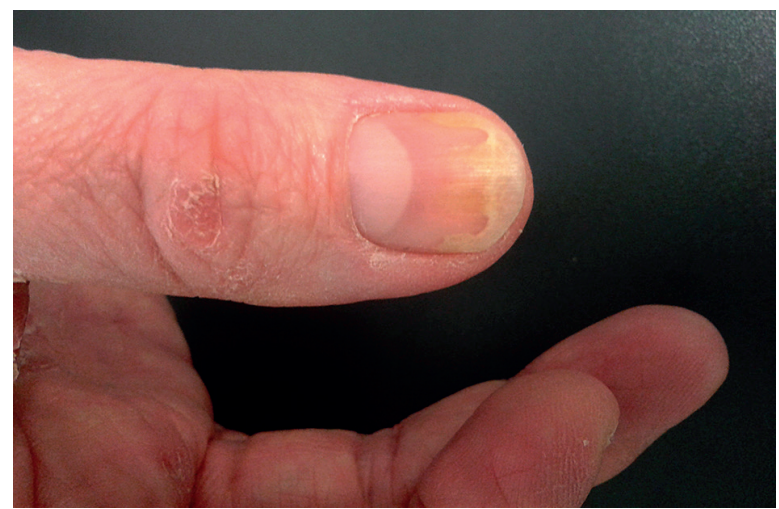

Fig. 2. Onycholysis and oil drops visible in the nail bed.

the lunula. For distal nail matrix PGA the total number of lesions in the distal nail plate is evaluated: clear if there are 2 or fewer leukonychia or other defects other than pits, almost clear if there are 1 to 5 pits or leukonychia, mild 6 to 10 , moderate 11 to 20 , severe 21 or more pits, leukonychia or crumbling. PGA nail bed assesses the $\%$ affected area: 0 means clear, i.e. when the free edge of the nail plate conforms to the arcuate shape of the distal groove, 1 (almost clear) means 10\% or less of the area is changed by onycholysis and/or salmon patches, 2 (mild) means 11 to 25\%, 3 (moderate) means 26-50\%, and 4 (severe) means that $51 \%$ or more of the nail bed is affected any onycholysis and/or salmon patches [15].

Ultrasound is considered a good tool to evaluate the different components of psoriatic nails $[16,17]$.

Skin activity in psoriasis is graded by the Psoriasis Area and Severity Index (PASI). PASI is a quantitative rating scale for measuring the severity of psoriatic lesions based on area coverage and plaque appearance. The plaques are characterized by erythema, thickness and scaling. Each is rated from 0 to 4, where 0 is none, 1 slight, 2 moderate, 3 severe, and 4 very severe. Each of the three 
plaque characteristic features is rated for each body region: head, upper limbs, trunk, and lower limbs. The scores for erythema, thickness and scaling for each body region are added together to give 4 separate subtotals. Next they are multiplied by the value of the body surface area represented by each region. The subtotal for the head is multiplied by 0.1 , upper limbs 0.2 , trunk 0.3 , and lower limbs 0.4. Then the degree of involvement as the percentage for each body region is scored from 0 to 6 . A score of 0 is none, 1 is $1-9 \%, 2$ is $10-29 \%, 3$ is $30-49 \%$, 4 is $50-69 \%, 5$ is $70-89 \%$, and 6 is $90-100 \%$. Then for each body region the previously counted subtotals are multiplied by the degree of involvement score (0-6). The PASI score (0-72) is the sum of the added 4 subtotals.

Body surface area (BSA) in \% means the percentage of patients' skin affected by psoriatic lesions.

In 2015 the Comprehensive Assessment of the Psoriasis Patient (CAPP), a novel disease severity measure to more accurately assess the full burden of plaque psoriasis and subtypes, including inverse, scalp, nail, palmoplantar, and genital psoriasis, was introduced [18].

Moll and Wright described five clinical patterns among patients with PSA: distal interphalangeal (DIP), asymmetrical oligoarticular, symmetric polyarticular, spondylitis, and arthritis mutilans [19]. In PsA asymmetric joints including distal interphalangeal joints (DIP) are often affected. They are evaluated for swelling and tenderness. GRAPPA recommended that the ACR joint count of 68 tender and 66 swollen joints count be used in PsA, and it can be readily performed in a clinic visit [20]. It was decided not to include the distal joints of the feet as it may be difficult to distinguish proximal interphalangeal (PIP) joint from DIP joint inflammation in the toes.

There are numerous tools to evaluate patients with PsA: the Toronto Psoriatic Arthritis Screening Questionnaire (TOPAS), the Psoriasis Epidemiology Screening Tool (PEST), the Psoriatic Arthritis Screening and Evaluation (PASE), the Psoriasis and Arthritis Screening Questionnaire (PASQ Disease Activity index for Psoriatic Arthritis (DAPSA), the Psoriatic Arthritis Joint Activity Index (PSAJAI), and the Composite Psoriatic Disease Activity Index (CPDAI) [21].

In the study of Lai et al. nail psoriasis was the most significant associated factor of DIP arthritis. Crumbling and onycholysis were found to be significantly associated with DIP disease [22]. However, sometimes it is extremely difficult to distinguish PsA (especially if it is sine psoriasis) from rheumatoid arthritis (RA). Dermoscopy may be a useful supportive tool for differentiating early PsA from RA [23].

Nail psoriasis is considered one of the most difficult forms of psoriasis to treat. Topical agents - corticostero- ids, vitamin A and D derivatives tazarotene, fluorouracil, dithranol, topical cyclosporine and topical psoralen UV-A - were used particularly in mild cases [5]. Conventional systemic treatments, including methotrexate, cyclosporine, acitretin, and apremilast, as well as intralesional corticosteroids, can also be effective treatments for nail psoriasis. The available evidence suggests that all anti-tumor necrosis factor- $\alpha$, anti-interleukin 17 , and anti-IL-12/23 antibodies which are available for plaque psoriasis and psoriatic arthritis are highly effective treatments for nail psoriasis [24, 25].

\section{Summary}

In conclusion, rheumatologists apart from joint assessment should also pay attention to the nail appearance in their patients. Nail psoriasis and/or psoriatic lesions in the gluteal cleft and on the scalp are sometimes the only features of psoriasis and are strongly correlated with PsA, especially in adults.

The author declares no conflict of interest.

\section{References}

1. Schons KR, Knob CF, Murussi N, et al. Nail psoriasis: a review of the literature. An Bras Dermatol 2014; 89: 312-317.

2. Elkayam O, Ophir J, Yaron M, et al. Psoriatic arthritis: interrelationships between skin and joint manifestations related to onset, course and distribution. Clin Rheumatol 2000; 19: 301-305.

3. Williamson L, Dalbeth N, Dockerty JL, et al. Extended report: nail disease in psoriatic arthritis - clinically important, potentially treatable and often overlooked. Rheumatology 2004; 43: 790-794.

4. Pourchot D, Bodemer C, Phan A, et al. Evaluation in 313 Children with Psoriasis. Pediatr Dermatol 2016 22. doi: 10.1111/ pde.13028.

5. Sánchez-Regaña M, Umbert P. Diagnosis and management of nail psoriasis. Actas Dermosifiliogr 2008; 99: 34-43.

6. Garbers LE, Slongo H, Fabricio LH, et al. Incidence, clinical manifestations and clipping of nail psoriasis in the dermatology center of the Hospital Universitário Evangélico de Curitiba. An Bras Dermatol 2016; 91: 300-305.

7. Balestri R, Rech G, Rossi E, et al. Natural history of isolated nail psoriasis and its role as a risk factor for the development of psoriatic arthritis: a single center cross sectional study. $\mathrm{Br}$ J Dermatol 2016; doi: 10.1111/bjd.15026.

8. Lawry M, Rich P. The nail apparatus: A guide for basic and clinical science. Curr Probl Dermatol 1999; 11: 163-208.

9. Wiland P. Zmiany narządowe $w$ chorobach reumatycznych. Górnicki Wydawnictwo Medyczne, Wrocław 2016.

10. Rich P, Scher RK. Nail Psoriasis Severity Index: A useful tool for evaluation of nail psoriasis. J Am Acad Dermatol 2003; 49: 206-212.

11. Parrish CA, Sobera JO, Elewski BE. Modification of the Nail Psoriasis Severity Index. J Am Acad Dermatol 2005; 53: 745-746. 
12. Baran RL. A nail psoriasis severity index. Br J Dermatol 2004; 150: 568-569.

13. Cassell SE, Bieber JD, Rich P, et al. The modified Nail Psoriasis Severity Index: validation of an instrument to assess psoriatic nail involvement in patients with psoriatic arthritis. J Rheumatol 2007; 34: 123-129.

14. Castellanos-González M, Joven BE, Sánchez J, et al. Nail involvement can predict enthesopathy in patients with psoriasis. J Dtsch Dermatol Ges 2016; 14:1102-1107.

15. Wong PCH, Leung YY, Li EK, et al. Measuring Disease Activity in Psoriatic Arthritis. Int J Rheumatol 2012; 2012: 839425.

16. Acquitter M, Misery L, Saraux A, et al. Detection of subclinical ultrasound enthesopathy and nail disease in patients at risk of psoriatic arthritis. Joint Bone Spine 2016; pii: S1297319X(16)30198-1.

17. Gutierrez M, Kaeley GS, Bertolazzi C, et al. State of the art of ultrasound in the assessment of psoriasis and psoriatic arthritis. Expert Rev Clin Immunol 2016; doi: http://dx.doi.org/10.10 80/1744666X.2017.1262765.

18. Paek SY, Thompson JM, Qureshi AA, et al. Comprehensive Assessment of the Psoriasis Patient (CAPP): A Report from the GRAPPA 2015 Annual Meeting. J Rheumatol 2016; 43: 961-964.

19. Moll JM, Wright V. Psoriatic arthritis. Semin Arthritis Rheum 1973; 3: 55-78.
20. Gladman DD, Mease PJ, Healy P, et al. Outcome measures in PsA. J Rheumatol 2007; 8: 1159-1166.

21. Raychaudhuri SP, Wilken R, Sukhov AC, et al. Management of psoriatic arthritis: Early diagnosis, monitoring of disease severity and cutting edge therapies. J Autoimmun 2017; 76: 21-37.

22. Lai TL, Pang HT, Cheuk YY, et al. Psoriatic nail involvement and its relationship with distal interphalangeal joint disease. Clin Rheumatol 2016; 35: 2031-2037.

23. Errichetti E, Zabotti A, Stinco G, et al. Dermoscopy of nail fold and elbow in the differential diagnosis of early psoriatic arthritis sine psoriasis and early rheumatoid arthritis. J Dermatol 2016; 43: 1217-1220.

24. Pasch MC. Nail psoriasis: a review of treatment options. Drugs 2016; 76: 675-705.

25. Torii H, Nakano M, Yano T, et al. SPREAD Study Group Efficacy and safety of dose escalation of infliximab therapy in Japanese patients with psoriasis: Results of the SPREAD study. J Dermatol 2016; doi: 10.1111/1346-8138.13698. 